\title{
Article \\ Computational Thermal Analysis of the Photothermal Effect of Thermoplasmonic Optical Fiber for Localized Neural Stimulation In Vivo
}

\author{
Woongki Hong (D), Junhee Lee, Duhee Kim, Yujin Hwang, Hyuk-Jun Kwon (D), Jae Eun Jang and Hongki Kang *(D) \\ Department of Information and Communication Engineering, Daegu Gyeongbuk Institute of Science and \\ Technology DGIST, Daegu 42988, Korea; monsterinc@dgist.ac.kr (W.H.); junhee@dgist.ac.kr (J.L.); \\ travelkim@dgist.ac.kr (D.K.); huj1804@dgist.ac.kr (Y.H.); hj.kwon@dgist.ac.kr (H.-J.K.); jang1@dgist.ac.kr (J.E.J.) \\ * Correspondence: hkang@dgist.ac.kr; Tel.: +82-53-785-6328
}

Citation: Hong, W.; Lee, J.; Kim, D.; Hwang, Y.; Kwon, H.-J.; Jang, J.E.; Kang, H. Computational Thermal Analysis of the Photothermal Effect of Thermoplasmonic Optical Fiber for Localized Neural Stimulation In Vivo. Electronics 2021, 10, 118. https:// doi.org/10.3390/electronics10020118

Received: 1 December 2020

Accepted: 6 January 2021

Published: 8 January 2021

Publisher's Note: MDPI stays neutral with regard to jurisdictional clai$\mathrm{ms}$ in published maps and institutional affiliations.

Copyright: (C) 2021 by the authors. Licensee MDPI, Basel, Switzerland. This article is an open access article distributed under the terms and conditions of the Creative Commons Attribution (CC BY) license (https:// creativecommons.org/licenses/by/ $4.0 /)$.

\begin{abstract}
Optical neuromodulation is a versatile neural stimulation technology that enables highly localized excitatory or inhibitory stimulation of neuronal activities. Photothermal neural stimulation using thermoplasmonic metallic nanoparticles for light to heat conversion has been suggested as an optical neural stimulation technology without genetic modification. Optical fibers implementing the thermoplasmonic effect were recently developed for localized neural stimulation, and the successful demonstration of localized neural stimulation in vitro was reported. However, before photothermal neural stimulation is further applied in the brains of live animals and ultimately in human trials, a safety analysis must carefully be performed for the thermal effect of stimulation in vivo. With the complexity of the physical structure and different thermal properties of the brain and surrounding body, the resulting thermal effect could vary despite the same power of light delivered to the optical fiber. In addition, dynamic thermal properties of the brain such as the daily blood perfusion rate change or metabolic heat generation must also be carefully considered for the precise implementation of photothermal neural stimulation. In this work, an in-depth computational analysis was conducted of the photothermal effects using a thermoplasmonic optical fiber for in vivo neural stimulation. The effects of the experimental design and stimulation protocols on the thermal effect in the brain were analyzed. We believe that the results provide a good experimental guideline for safely conducting photothermal neural stimulation using the thermoplasmonic optical fiber technology.
\end{abstract}

Keywords: optical neuromodulation; neural stimulation; photothermal effect; thermoplasmonics; finite-element analysis (FEA)

\section{Introduction}

The localized photothermal effect using thermoplasmonic metal nanoparticles has great potential in biomedical engineering. Upon the illumination of light with a specific wavelength, plasmonic nanoparticles efficiently convert the optical energy to heat energy, and the nanoparticles operate as a collective heat source to increase the temperature of their surroundings [1-3]. This photothermal effect has been widely applied to biomedical applications, including photothermal tumor and cancer therapy [4-6]. In addition to these applications, the photothermal effect has been recently suggested for the optical modulation of brain activities [7,8]. Conventional electrical neural stimulation shows only an excitatory stimulation effect of the stimulated neurons. It is difficult to localize the stimulation effect because the electrical current flows inside the brain. However, optical neural stimulation such as optogenetics has shown that the stimulated neurons can be either excited or inhibited depending on the experimental protocols. Moreover, the optical stimulation effect can be highly localized by confining the illuminated light [9-11].

Among the various optical neuromodulation technologies, photothermal stimulation using thermoplasmonic metal nanoparticles has shown versatile functionalities (i.e., excitation and inhibition) without the need for genetic modification. The combination of 
tissue-transparent near-infrared (NIR) light (adsorption coefficient of 0.3 in the brain) [12] and NIR-sensitive plasmonic nanoparticles as the conversion agent from light energy to heat energy through the localized surface plasmonic resonance (LSPR) enables a localized temperature increase and precise control of neural activity $[13,14]$. Among the several suggested biophysical mechanisms for the neuromodulation, the generated heat from the plasmonic nanoparticles is delivered to the plasma membrane of the target neurons and induces neural stimulation by either activating thermally sensitive endogenous potassium channels on the membranes or modifying the plasma membrane capacitances. In the investigation of neural circuits in the brain and potential treatments for neurological disorders by photothermal stimulation, many different engineering approaches have been studied to implement the thermoplasmonic effect and verify the stimulation effects. As examples of in vitro neural stimulation, photothermal neural stimulation for single-cell resolution $[15,16]$, light patterning and nanoparticle patterning for selective neuromodulation $[17,18]$ have been demonstrated.

In addition, thermoplasmonic gold nanorods attached to optical fiber have been recently developed for localized photothermal neuromodulation for both in vitro and in vivo neuronal networks [19]. Simple fabrication methods for plasmonic nanoparticle attachment easily converted commercial optical fiber cannulas to a photothermal local stimulation tool. Because optical fiber cannulas have been routinely used in optogenetics as some of the standardized stimulation protocols, the application of this new technology to neuroscientific research is promising. As evidence of the potential of this new technology, localized neural stimulation using a thermoplasmonic optical fiber was demonstrated in vitro on hippocampal neuronal networks in the same work. Despite the slightly higher power of the NIR laser used for the thermoplasmonic effect (24-50 $\mathrm{mW}$ in vitro) compared with the visible lights used in optogenetics, the reduced scattering attenuation of the long-wavelength NIR is expected to be important for reducing photodamage.

In this study, we analyzed the spatiotemporal heat transfer characteristics when a thermoplasmonic optical fiber is used for various in vivo neural stimulation environments (Figure 1). For the safe implementation of the photothermal neural stimulation in the brains of live animals and ultimately in human trials, we must carefully analyze the thermal effect of stimulation in vivo and execute the light illumination accordingly. With the complexity of the physical structure and different thermal properties of the brain and surrounding body (e.g., skull or scalp), the resulting thermal effect could vary despite the same power of light delivered to the optical fiber. For the precise estimation of heat transfer, we used finiteelement method (FEM) simulation, which mimics realistic experimental environments. We analyzed the spatiotemporal temperature changes of the stimulated area in the brain with potential in vivo neural stimulation conditions, with and without covering the brain by the skull and scalp. Furthermore, we analyzed the influence of metabolic heat generation and the blood perfusion rate on the photothermal effects. The dynamic thermal properties of the brain such as daily cerebral blood flow change or metabolic heat generation must also be carefully considered for more precise implementation of long-term neural stimulation in the brain, especially for behavior studies. 


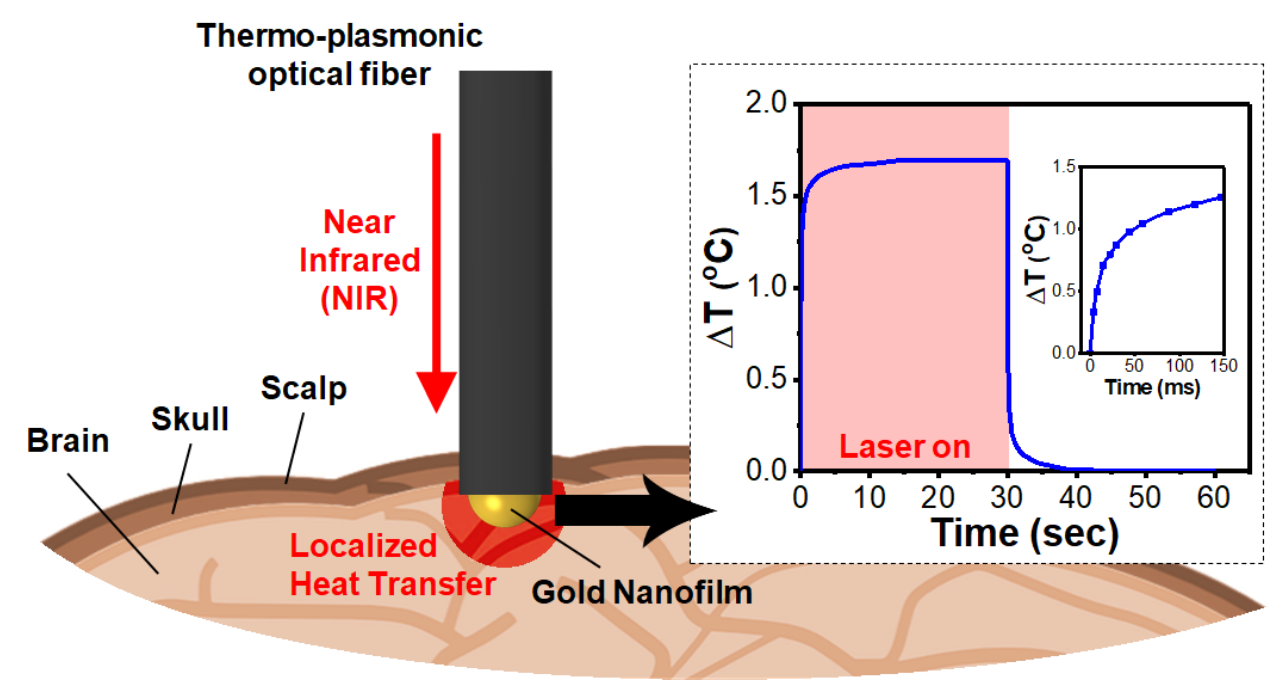

Figure 1. Schematic illustration of in vivo photothermal stimulation by a thermoplasmonic optical fiber. The inset image shows the temperature changes by the thermoplasmonic optical fiber.

\section{Experimental Setup}

2.1. Simulation Model Design of the In Vivo Neural Stimulation Using a Thermoplasmonic Optical Fiber

Thermal analysis of photothermal stimulation by the thermoplasmonic optical fiber was performed on COMSOL Multiphysics v5.5a (COMSOL Inc., Burlington, MA, USA). In the previously published experimental neural stimulation work, the following laser and optical fiber setup was used. Through a multimode optical fiber with a core diameter of $200 \mu \mathrm{m}$, continuous-wave diode-laser-illuminated near-infrared-light was used (785 nm). For inhibitory neural stimulation, light illumination was applied continuously for a certain duration of time (e.g., tens of seconds) to suppress the neuronal activities during stimulation. To create similar environments with the in vivo experiments, the optical fiber was made to penetrate a surrounding block that consisted of the brain with a volume of $20 \times 20 \times 10 \mathrm{~mm}^{3}$ (Figure 2a). The penetrated optical fiber was modeled based on a semi-sphere-shaped termination design taken from a previous work, which showed the highest photothermal efficiency [19]. The core of the optical fiber (silica glass) had a $200 \mu \mathrm{m}$ diameter with a variable length from 0 to $3.4 \mathrm{~mm}$, depending on the penetration depth into the brain. It was surrounded by a $100 \mu \mathrm{m}$ thick cladding of PMMA material. At the end of the fiber, the fiber optic cannula made of silica glass was attached, which was $1.6 \mathrm{~mm}$ in diameter and $4 \mathrm{~mm}$ in thickness [19]. In the previously published thermoplasmonic optical fiber work, owing to the strong thermoplasmonic effect of rod-shaped gold nanoparticles at NIR, gold nanorods (approximately $15 \mathrm{~nm} \times 45 \mathrm{~nm}$ ) were attached to the optical fiber [19]. However, in this computational analysis, we did not implement such small nanostructures due to the computation limit. Instead of having gold nanorods as the thermoplasmonic layer, we defined the thermoplasmonic layer as a continuous gold layer that had a $500 \mathrm{~nm}$ thickness coated on the core tip of the fiber (Figure 2b). Though we defined the nanostructure of gold, the gold layer structure itself was not intended to mimic the nanoplasmonic characteristics. The heat rate in $\mathrm{mW}$ was directly applied to the gold layer to mimic the thermoplasmonically converted heat from light. By taking this simplified approach, we were able to significantly reduce the computations in our simulation for the transient response of the thermoplasmonic effect. 
a

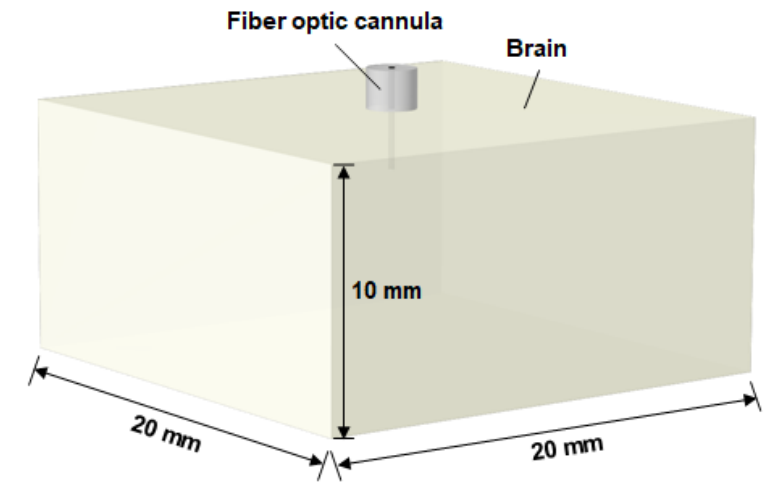

c

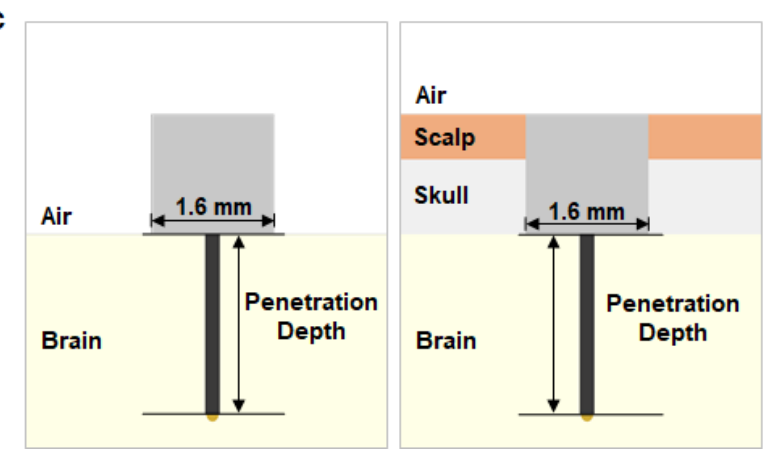

b

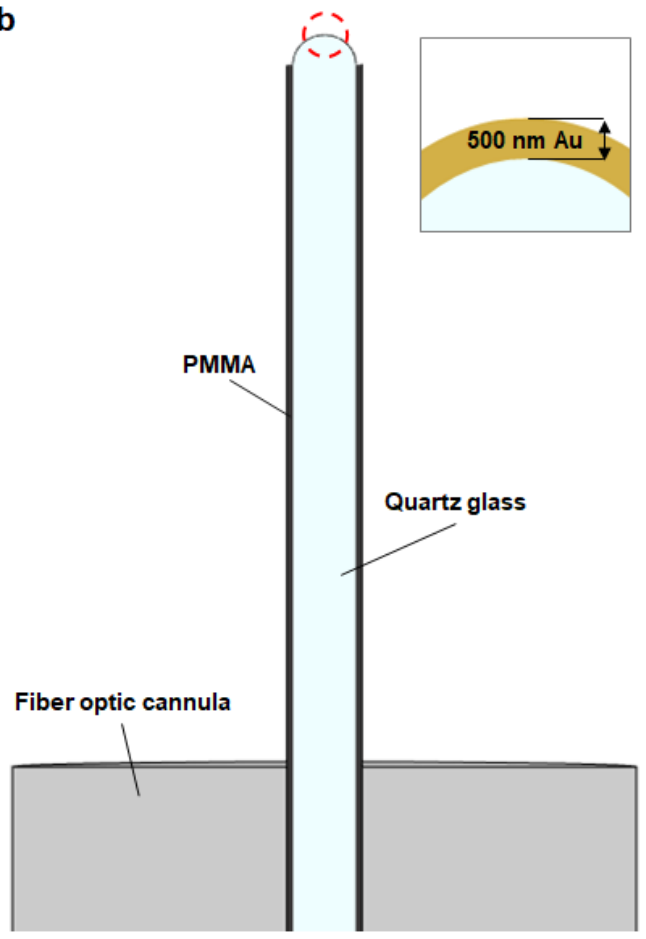

Figure 2. Computational model of the temperature analysis of the photothermal effect using TP optical fiber in vivo. (a) Schematic graphics of gold-nanorods-attached optical fiber in the brain for the finite-element analysis (FEA). (b) Crosssectional view of the optical fiber and brain without and with the skull and scalp. (c) Model of gold-nanorods-attached optical fiber. Inset image shows the thickness of the gold nanofilm coated on the core tip of the optical fiber.

Based on the aforementioned dimensions, this study considered two different models to study the difference in heat transfer on the brain by the various surrounding materials: the brain exposed to air and the brain covered by the skull and scalp [20] (Figure 2c). The thicknesses of the skull and scalp were set to $3 \mathrm{~mm}$ and $1 \mathrm{~mm}$, respectively. We did not consider precise geometrical properties such as the curvature of the brain, skull and scalp due to the significantly different dimensions that led to convergence failures in the simulations.

\subsection{Computational Analysis of Spatiotemporal Heat Transfer in the Brain}

We simulated spatiotemporal heat transfer characteristics using a heat transfer module called the bioheat transfer module, provided by COMSOL. Using this module, we analyzed the precise temperature change around the optical fiber in the brain based on heat conduction and biological elements such as blood perfusion rate and metabolic heat generation. To simulate heat generation from the gold layer, a boundary heat source was applied to the external surface of the gold layer. Bioheat transfer analysis was performed based on the following Pennes bioheat equation [21]:

$$
\rho C_{p} \frac{\partial T}{\partial t}=k \nabla^{2} T+\eta_{b} \rho_{b} C_{p b}\left(T_{a}-T\right)+Q_{m e t}
$$

where $\rho, C_{p}, k, \eta_{b}$ and $Q_{m e t}$ are the mass density $\left(\mathrm{kg} / \mathrm{m}^{3}\right)$, specific heat capacity $(\mathrm{J} / \mathrm{kg} \cdot \mathrm{K})$, thermal conductivity $(\mathrm{W} / \mathrm{m} \mathrm{K})$, blood perfusion rate $(1 / \mathrm{s})$ and volumetric metabolic heat generation rate of the tissue $\left(\mathrm{W} / \mathrm{m}^{3}\right)$, respectively. $\rho_{b}, C_{p b}$ and $T_{a}$ are the blood density $\left(\mathrm{kg} / \mathrm{m}^{3}\right)$, specific heat capacity of blood $(\mathrm{J} / \mathrm{kg} \cdot \mathrm{K})$ and temperature of the arterial blood $\left({ }^{\circ} \mathrm{C}\right)$, respectively. Because the maximum temperature change is small $\left(<10^{\circ} \mathrm{C}\right)$, we did not include heat convection. Furthermore, we set the blood perfusion rate to various values to 
analyze the change of heat transfer by the blood perfusion rate. The material properties applied in this simulation are listed in Table 1.

Outside the brain, when the brain is exposed to air, we assumed that inward heat flux occurred over a boundary, which is exposed to air with the following equation [22]:

$$
q_{0}=h\left(T_{\text {ext }}-T\right)
$$

where $h$ and $T_{\text {ext }}$ are the heat transfer coefficient and external temperature, respectively. We assumed the initial temperature of the brain to be $37^{\circ} \mathrm{C}$, the external air temperature to be $24^{\circ} \mathrm{C}$ and the heat transfer coefficient $(h)$ to be $5 \mathrm{~W} /\left(\mathrm{m}^{2} \cdot \mathrm{K}\right)$ [20]. To maximize the simulation accuracy, we set the relative tolerance and maximum number of iterations to $10^{-6}$ and 25 , respectively.

Table 1. Material properties applied in the computational simulation $[19,20,23]$.

\begin{tabular}{|c|c|c|c|c|c|}
\hline Materials & $\begin{array}{l}\text { Density } \\
\left(\mathrm{kg} / \mathrm{m}^{3}\right)\end{array}$ & $\begin{array}{l}\text { Heat Capacity } \\
(\mathrm{J} / \mathrm{kg} \cdot \mathrm{K})\end{array}$ & $\begin{array}{l}\text { Thermal Conductivity } \\
(\mathrm{W} / \mathrm{m} \cdot \mathrm{K})\end{array}$ & $\begin{array}{c}\text { Blood Perfusion Rate } \\
(1 / \mathrm{s})\end{array}$ & $\begin{array}{c}\text { Metabolic Heat } \\
\text { Generation }\left(\mathrm{W} / \mathrm{m}^{3}\right)\end{array}$ \\
\hline Silica glass & 2500 & 742 & 1.37 & - & - \\
\hline Air & 1.188 & 1006 & 0.02617 & & \\
\hline Brain & 1041 & 3640 & 0.528 & $0.003-0.0154$ & 10,383 \\
\hline Skull & 1990 & 1300 & 0.65 & 0.00099 & 26 \\
\hline Scalp & 1100 & 3150 & 0.342 & 0.0022 & 1100 \\
\hline Blood & 1060 & 3840 & 0.530 & - & - \\
\hline Gold & 19,320 & 125.604 & 314 & - & - \\
\hline PMMA & 1.18 & 1466 & 0.2085 & - & - \\
\hline
\end{tabular}

\subsection{Experimental Validation of the Numerical Model}

For the experimental validation of the numerical model, we adopted the relationship between the experimental results and the simulation results from a previous work [19]. The thermoplasmonic optical fiber model defined in the simulation in the previous section is identical to the model used in the previous work. In the previous work, the temperature change at the tip of the optical fiber upon light illumination in the numerical simulation was directly compared with a thermographic infrared camera measurement in the same surrounding conditions. The simulation model quite accurately demonstrated the temporal characteristics of the thermoplasmonic effect by the optical fiber. We used the heat rate $(\mathrm{mW})$ as an input for the numerical simulation, and laser power $(\mathrm{mW})$ as an input for the photothermal experiment. Therefore, in the previous work, the relationship between the NIR laser power $(\mathrm{mW})$ and the rate $(\mathrm{mW})$ was experimentally obtained as follows: heat rate $(\mathrm{mW})$ /laser power $(\mathrm{mW})=15.8 \%$. Moreover, we used the conversion information as an experimental validation of the numerical model in this simulation work [19].

\section{Results}

In vivo photothermal neural stimulation using a thermoplasmonic optical fiber can in many aspects follow well-established experimental optogenetics protocols. The most common implant procedure would be the following. First, a selected virus is injected into an area of interest in the brain. Second, an optical fiber is implanted into the brain, and the length of the optical fiber is determined based on the depth of the region of interest from the top surface of the brain. During implantation, a single microelectrode or microelectrode array is implanted along with the optical fiber for the electrical recording of a single neuron or neuronal population. Lastly, after a period of time of days or weeks for the recovery of the animal and the expression of the optogenetic agents' opsins, photostimulation and simultaneous electrical recording can be performed [9]. The biggest modification for photothermal stimulation from the optogenetics protocol would be that the virus transfection period is not required. Therefore, there is no waiting time necessary for the expression of opsins. Hence, the experimental procedure will be much more simplified, 
and acute recording and stimulation can be performed immediately after the insertion of the thermoplasmonic optical fiber and microelectrodes.

According to this difference, we should consider both acute and long-term cases in in vivo photothermal neural stimulation experimental conditions. In other words, the thermal effects in the brain upon photothermal stimulation need to be analyzed with both the brain exposed to air, which mimics more closely the acute experiment, and the brain closed from the outside after fiber implantation, which mimics long-term experiments [24,25]. Therefore, in this work, we considered two cases of in vivo photothermal stimulation experimental conditions depending on whether the skull is open or not. In those conditions, we conducted a computational simulation of the thermal effect by finite-element method (FEM) described in the Experimental Setup section (Section 2) for the aforementioned experiments. We then analyzed the spatiotemporal characteristics of the temperature change around the implanted thermoplasmonic optical fiber in the brain. In the following sections, we provide the simulation results and discuss the implications.

\subsection{Thermal Analysis of Photothermal Stimulation When the Brain Is Exposed to Air}

We first simulated the heat transfer of the thermoplasmonic optical fiber in the brain exposed to air. As explained in the previous section, the aim was to mimic a photothermal stimulation environment condition in the brain of a live animal while the brain was exposed to air [10]. Acute implantation of the optical fiber was therefore mimicked. Figure 3a shows the representative simulation result of the thermoplasmonic effect based on the optical fiber in the brain (penetration depth of $1.4 \mathrm{~mm}$ ). As shown in the temperature change profile, a significant temperature change occurred around the tip of the hemispherical optical fiber tip, and the thermoplasmonic effect was highly localized. The generated heat from the gold nanofilm layer was locally transferred at a diameter of $1 \mathrm{~mm}$, and the peak temperature increase was proportional to the implemented heat rate $(\mathrm{mW})$ at $1.732 \pm 0.026^{\circ} \mathrm{C} / \mathrm{mW}$ (Figure $3 \mathrm{~b}$ ). Considering the equivalent light power $(\mathrm{mW})$ for the heat rate (see Experimental Setup (Section 2) for more details), the peak temperature increase was also proportional to the NIR laser power $(\mathrm{mW})$ with the following relationship: $0.27 \pm 0.03{ }^{\circ} \mathrm{C} / \mathrm{mW}$. As shown in Figure $3 c, d$, the temperature change of the brain by the thermoplasmonic effect during heat-up reaches a steady-state condition and cools down to the initial body temperature in approximately $500 \mathrm{~ms}$. The spatiotemporal characteristics observed from the simulation results are similar to the localized neural stimulation experimental results in a previous work: hundreds of $\mu \mathrm{m}$ in the effective area and hundreds of $\mathrm{ms}$ in the timescale [19].
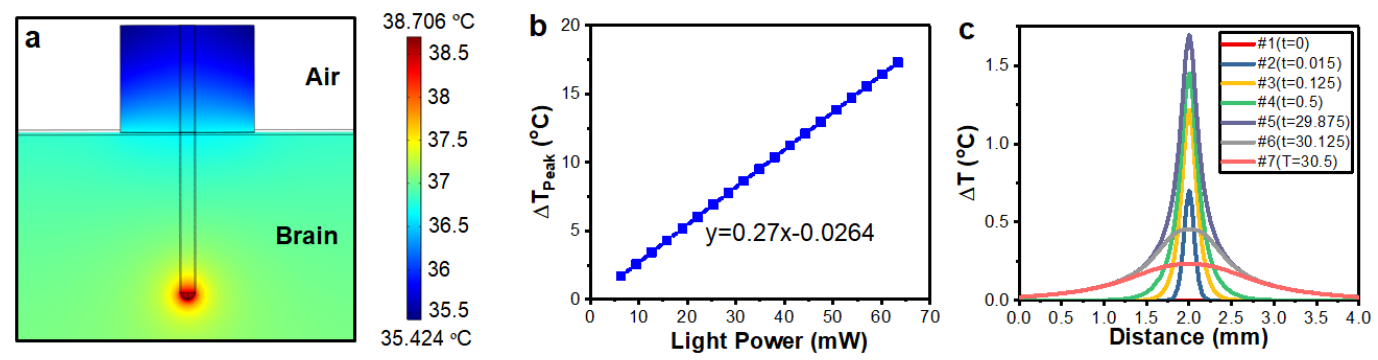

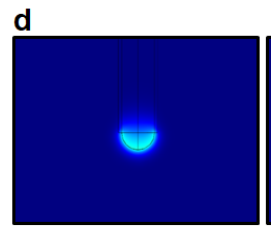

$t=0.015 \mathrm{sec}$

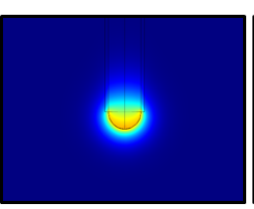

$\mathrm{t}=0.12 \mathrm{sec}$

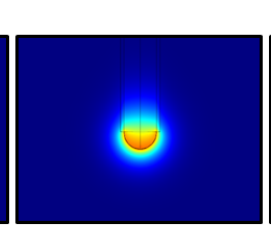

$\mathrm{t}=0.25 \mathrm{sec}$

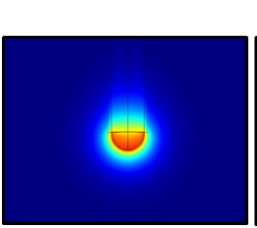

$\mathrm{t}=0.4 \mathrm{sec}$

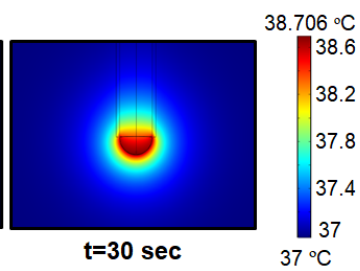

Figure 3. Simulation results of the temporal temperature changes of the TP optical fiber. (a) Simulation results of the temperature changes on the brain (penetration depth: $1.4 \mathrm{~mm}$, heat rate: $1 \mathrm{~mW}$, equivalent laser power: $15.8 \mathrm{~mW}, 30 \mathrm{~s}$ ). (b) Peak temperatures of the brain under light power increases. (c,d) One-dimensional (1D) and 2D plots of temporal temperature changes of the brain by photothermal stimulation (penetration depth: $1.4 \mathrm{~mm}$; heat rate: $1 \mathrm{~mW}$; equivalent laser power: $15.8 \mathrm{~mW}$; $40 \mathrm{~s}$ with $30 \mathrm{~s}$ of laser irradiation). 
Using the computational model above, we then analyzed the effect of the position of the stimulation target by varying the penetration depth of the thermoplasmonic optical fiber. Despite using the same stimulation laser power conditions, the temperature profiles of the brain around the optical fiber for the different penetration depths were significantly different (Figure 4). Peak temperature changes from the profiles in Figure 4 are plotted in Figure 5a. When photothermal stimulation was applied on the surface of the brain (smaller penetration depth), the peak temperature change was comparably much higher at $2.47^{\circ} \mathrm{C}$. However, the peak temperature change drastically decreased as the penetration depth increased. Eventually, the peak temperature change was saturated at around $1.7^{\circ} \mathrm{C}$ after penetrating over $1 \mathrm{~mm}$ from the surface (Figure 5a). Therefore, when the brain surface is photothermally stimulated, up to a $45 \%$ more temperature change is likely to occur, and the change of the temperature is very sensitive to the penetration depth.

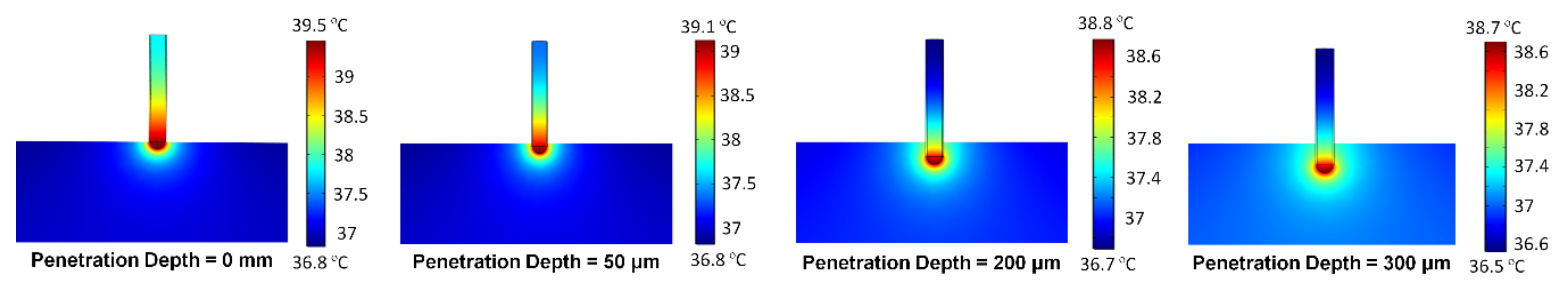

Figure 4. Computational analysis of the temperature changes of the TP optical fiber with different penetration depths (heat rate: $1 \mathrm{~mW}$; equivalent laser power: $15.8 \mathrm{~mW} 30 \mathrm{~s}$ after the laser was turned on).

a

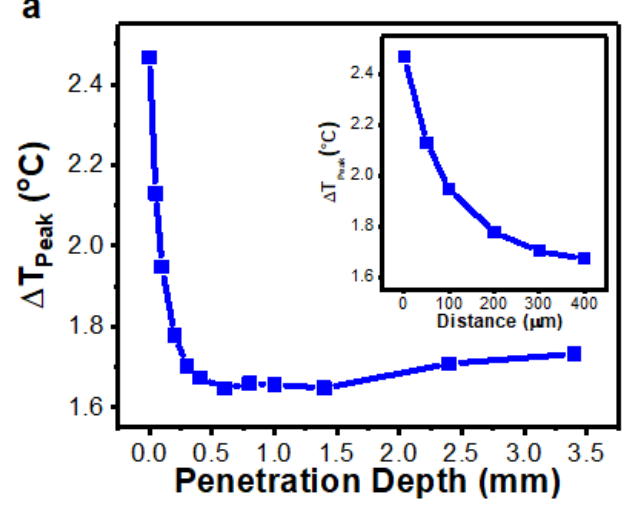

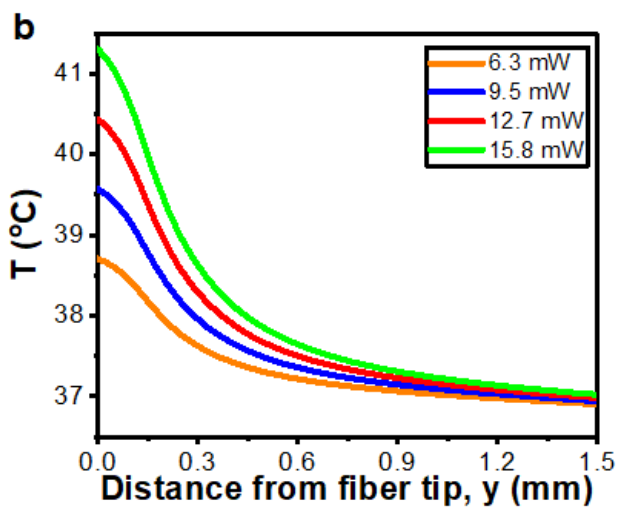

Figure 5. Spatial temperature analysis of the TP optical fiber in the brain. (a) Temperature changes of the TP optical fiber with different penetration depths. Each light power was set to $6.3 \mathrm{~mW}$. (b) Temperature distribution of the TP optical fiber with a different light power $(\mathrm{mW})$. Penetration depth was set to $1.4 \mathrm{~mm}$.

Based on the simulation results in Figure 4, we think the significant differences are due to the much smaller thermal conductivity and heat capacity of the surrounding air compared with the characteristics of the brain. Because air is not an efficient thermal conductive material, the generated heat at the tip of the optical fiber must be diffused through either the brain or the fiber itself. When the fiber penetrates deeply (e.g., penetration depth: $300 \mu \mathrm{m}$ ), we see that significant heat diffusion occurs through the wide heat diffusion area of the brain surrounding the tip of the fiber. However, when the optical fiber is only placed on the surface of the brain, the fiber does not have a sufficient heat diffusion area due to the reduced contact with the brain, and thus, the heat is mostly confined at the tip of the fiber and the core material of the fiber. For the same amount of generated heat rate, the temperature change is higher. Therefore, it is necessary that we lower the laser power to implement the same results of photothermal stimulation on the brain surface.

In addition, we attempted to analyze the spatial temperature profile from the fiber tip along the penetration direction and the degree of localization of the thermoplasmonic effect under various heat rate conditions (Figure $5 b$ ). We observed that the temperature gradually 
decreased to $20 \%$ of the maximum temperature when around $500 \mu \mathrm{m}$ away from the fiber tip. Beyond $500 \mu \mathrm{m}$, the temperature slowly saturated to the baseline body temperature, which was defined at $37^{\circ} \mathrm{C}$ in our simulation.

\subsection{Thermal Analysis of Photothermal Stimulation When the Brain Is Covered by Skull and Scalp}

We further simulated the spatial profile of the temperature changes from the optical fiber when the brain is covered by the skull and scalp. This simulation condition represents the environments of typical long-term optical neural stimulation experiments. For example, behavior studies of live animals such as mice or rats are similarly performed with local neural stimulation using the optical fiber implanted into the target area of the brain. Figure $6 \mathrm{a}, \mathrm{b}$ exhibits representative simulation results of the temperature change profile around the implanted fiber (penetration depth: $0 \mathrm{~mm}$ for Figure $6 \mathrm{a}$ and $1.4 \mathrm{~mm}$ for Figure $6 \mathrm{~b}$ ). The temperature values along the y-axis were extracted and plotted in Figure $6 \mathrm{c}$, showing that the thermoplasmonic effect by the optical fiber is also similarly localized because it was observed in Figure $5 \mathrm{~b}$. It also shows the temperature change reduces to $20 \%$ of the peak temperature at $500 \mu \mathrm{m}$ from the fiber tip along the y-axis (Figure $6 \mathrm{~b}$ ). The most noticeable difference from the case when the brain was exposed to air (Figure 3) was that the temperature change profile was nearly insensitive to the penetration depth of the optical fiber. As shown in Figure $6 c, d$, despite the change in the penetration depth from 0 to $2.4 \mathrm{~mm}$, the temperature change profile was nearly unchanged. Even when the optical fiber was placed at the surface of the brain (penetration depth: $0 \mathrm{~mm}$ ), the optical fiber was not surrounded by air but by the skull and scalp. While the skull and the scalp are different from the brain in terms of thermal properties, the differences are relatively much smaller than in the case of air (Table 1). Therefore, instead of being influenced by the free-convection from air, when the brain is covered, the thermal characteristics of the optical fiber are less sensitive to the penetration depth of the fiber.
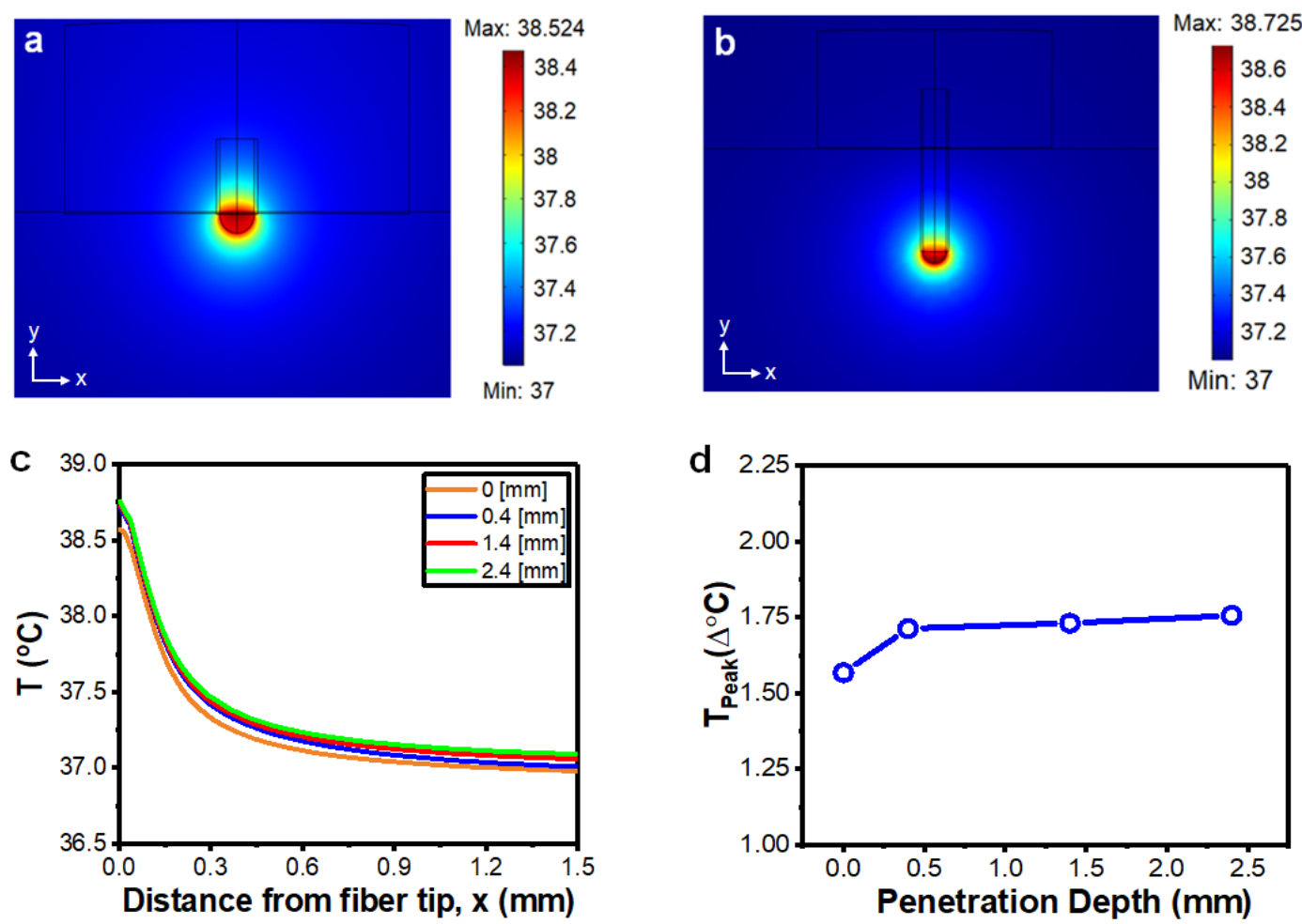

Figure 6. Temperature analysis of photothermal effect using TP optical fiber in the brain covered with the skull and scalp. $(\mathbf{a}, \mathbf{b})$ Top-view and cross-sectional view of the temperature distribution in the brain by the optical fiber. Penetration depths were set each to 0 and $1.4 \mathrm{~mm}$, and the heat rate was set to $1 \mathrm{~mW}$ for $20 \mathrm{~s}$. (c) Temperature distribution of the TP optical fiber with different penetration depths. (d) Temperature changes of TP optical fiber with different penetration depths (heat rate: $1 \mathrm{~mW}$; laser irradiation: $20 \mathrm{~s}$ ). 


\subsection{Effect of Cerebral Blood Flow and Metabolic Heat Generation}

Another important difference from the in vitro environment is that the brain of a live animal does not have a constant baseline temperature, but it shows daily temperature changes [26]. Therefore, to accurately predict the temperature change by photothermal stimulation in vivo, we must include two additional sources that can affect the heat transfer in the brain: cerebral blood flow and metabolic heat generation [20]. First, blood perfusion in the brain must be included, and the daily rhythm of the cerebral blood flow velocity must also be considered. Due to cerebral blood flow in the brain working as a heat sink, the generated thermal energy by the thermoplasmonic effect can be removed by the blood flow. As a consequence, the temperature change will vary. In addition, it has been reported that cerebral blood flow varies according to the sleep cycle and circadian rhythm [23]. With the popular applications of optical neuromodulation for long-term neurological behavioral studies or chronic neurological disorder treatment, the dynamic characteristics of the heat transfer characteristics in the brain must be carefully analyzed. Second, because the brain is an active metabolic organ, metabolic self-heat generation must also be considered in the computational analysis. In this section, we simulated the two mechanisms and analyzed the effect of photothermal stimulation using the thermoplasmonic effect.

Changes in the blood perfusion rate depend on sleep and wake processes. It starts to rise after awakening and reaches the peak rate in the late afternoon. The blood perfusion rate then decreases as it is entering into sleep periods [23]. For example, the blood perfusion rate can range from 0.003 to $0.015 \mathrm{~s}^{-1}$ for human trials [23]. Varying the blood perfusion rates, we further analyzed the influence of the changes in the blood perfusion rate and the metabolic heat generation (described as bioheat transfer in Figure 7). It is confirmed that the metabolic heat generation and blood perfusion rate influence the change in the photothermal effect and the localization of the resulting temperature change. When the two bioheat transfer mechanisms were considered, the peak temperature was increased by $0.344^{\circ} \mathrm{C}$ more, with a similar distribution for the $500 \mu \mathrm{m}$ distance from the fiber tip (Figure 7a). Compared with the simulation without the bioheat transfer mechanisms, $24.9 \%$ more temperature change is expected when both metabolic heat generation and blood perfusion are considered. The daily blood perfusion rate increases from $0.003 \mathrm{~s}^{-1}$ to $0.015 \mathrm{~s}^{-1}$ led to a peak temperature change ranging from $1.734-1.719{ }^{\circ} \mathrm{C}$ (Figure $7 \mathrm{~b}$ ). At best, only $0.12 \%$ of the peak temperature change was therefore observed. Lateral temperature distributions from the optical fiber tip with different blood perfusion rates were also almost similar because of a small temperature difference (Figure 7c).

a

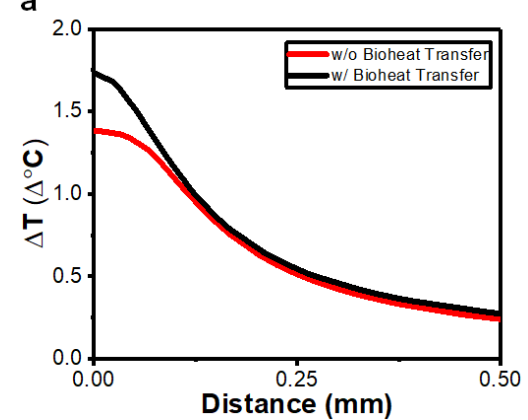

b

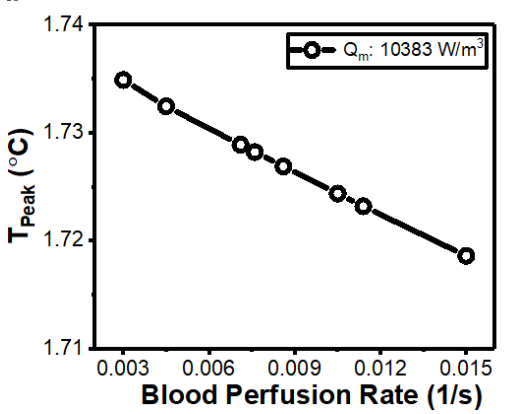

C

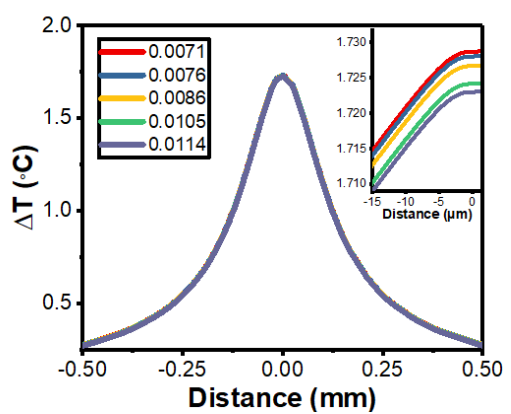

Figure 7. Temperature changes with various blood perfusion rates. (a) Thermal impacts of the metabolic heat generation and blood perfusion rate. (b) Temperature changes by the TP optical fiber with a change in the blood perfusion and metabolic heat generation of $10,383 \mathrm{~W} / \mathrm{m}^{3}$. (c) Temperature distribution by photothermal stimulation in the $x$-axis from the fiber tip under a various blood perfusion rates. Heat rate (equivalent laser power) and penetration depth were set to $1 \mathrm{~mW}$ (Equivalent light power: $15.8 \mathrm{~mW}$ ) and $1.4 \mathrm{~mm}$, respectively. 


\section{Discussion}

In this work, we performed an in-depth analysis of the thermal impacts of photothermal neural stimulation using a thermoplasmonic optical fiber. Before the experimental application of localized photothermal neural stimulation in vivo, the in-depth computational analysis of the thermal effects in the brain provided practically important experimental guidelines for the precise control of the temperature in various potential experimental conditions. With the guidelines in hand, we could avoid a situation where the temperature change was beyond what is required for neural stimulation, resulting in physical damage to the tissues. We could also provide a consistent application of the temperature change during stimulation for different surrounding environments.

We first confirmed that the computational model we utilized showed a localized temperature distribution in the brain, as previously published [19]. Furthermore, we compared two conditions of the photothermal neural stimulation experiments: one with the brain exposed to air and the other with the brain covered with the skull and scalp. We observed that these two conditions led to distinctively different spatial influences of the photothermal effect when the thermoplasmonic optical fiber was located near the surface of the brain. As summarized in Figure 4 when the penetration depth value was small (in other words, when we stimulated the surface of the brain), the temperature change was $45 \%$ $\left(0.81^{\circ} \mathrm{C}\right)$, higher than when the fiber was deeply inserted over $2.5 \mathrm{~mm}$. On the other hand, it demonstrated much more uniform temperature changes with different penetration depths when the brain was covered by the skull and scalp (Figure 6c). However, the temperature changes were similar for both cases when the fiber penetrated over a $2.4 \mathrm{~mm}$ depth.

As explained in the earlier sections, given the potential experimental conditions of photothermal stimulation using the thermoplasmonic optical fiber, the huge difference in temperature change we observed when the surface of the brain was stimulated while exposed to air is critically important. In the case of optogenetics, because light penetrates nearly identically toward the inside of the brain, regardless of the penetration depth of the fiber and the surrounding environments (i.e., either the skull is open or not), nearly the same light stimulation parameters (e.g., laser power and duration) can be maintained. However, for photothermal stimulation, because the generated heat can transfer in every direction in the brain, the thermal characteristics of the surrounding area would be critical in photothermal stimulation. Therefore, as we concluded from our simulation experiments, it is clear that we must significantly lower the laser power when we photothermally stimulate the surface of a brain exposed to air (e.g., acute experiment condition). This is because the surrounding air has much lower thermal conductivity than the brain, skull and scalp (Table 1). Therefore, the heat generated by the thermoplasmonic effect is used to increase the temperature of a much smaller volume of the brain compared to when the fiber penetrates deeply and thus has a much larger volume of the brain to heat. As a result, the temperature change is much higher when the brain is exposed to air. On the other hand, as shown in Figure 6d, when the brain was covered with the skull and scalp and the TP optical fiber stimulated the surface of the brain, a smaller temperature change was expected compared to the deeply penetrated optical fiber. From the different heat conductivities and thermal capacities of the skull and scalp from those parameters of the brain, a different spatial heat distribution occurred. Therefore, in this case, for the same thermal stimulation effect to occur, we will have to slightly increase the laser power.

In addition, in this work, we analyzed the effect of the daily change of thermal properties in the brain on the photothermal effect. Unlike other in vivo neural stimulations, we hypothesized that this dynamic behavior can present a distinct difference and must be considered to keep the stimulation effect consistent. As we analyzed in the previous section, when considering cerebral blood flow and metabolic heat generation effects, the temperature change by the photothermal effect is more localized than when not considered (Figure 7a). On the other hand, we observed that the daily change in cerebral blood flow had no significant effect on the change in temperature. In a general range of blood flow velocity under consistent metabolic heat generation, the peak temperature had a variation 
of only $1.4-1.7 \%$ of the overall temperature change. Therefore, the influence of the peak temperature variation on neural activity compared to photothermal inhibition is very small [27]. Therefore, we can anticipate that the photothermal neural stimulation using an optical fiber can be applied without significant illuminated laser power adjustment throughout the day. It is a clear benefit of using photothermal stimulation in vivo, especially for long-term behavioral stimulation experiments.

With the in-depth understanding from this work, photothermal stimulation can be applied more safely to neuroscience research and many neurological disorder treatment applications where localized excitatory and inhibitory stimulations are needed but virus transfection is not allowed due to long-term safety. As a next step, we anticipate that more in vivo localized photothermal neural stimulation studies will be carried out in order to validate the efficacy of this technology, as expected from in vitro experimental results. Furthermore, for less brain tissue damage during stimulation, reducing the diameter of the thermoplasmonic optical fiber can be considered as it has been pursued in optogenetics $[10,28]$. Compared with ultrasound-based neural stimulation, optical-fiber-based stimulation approaches are more invasive due to the insertion of microscale optical fiber. However, more precise targeting (submillimeter range) and more versatile stimulation effects (either excitatory or inhibitory stimulation) can be achieved with photothermal stimulation using thermoplasmonic optical fiber [29-31].

\section{Conclusions}

We performed an in-depth computational analysis of in vivo photothermal neural stimulation using a thermoplasmonic optical fiber. We confirmed that the thermal impacts of the optical fiber differed for different photothermal stimulation conditions. For the acute stimulation conditions when the brain was exposed to air, the temperature change strongly depended on the penetration depth of the fiber because of the limited areas of heat transfer and air convection. However, when the brain was covered with the skull and scalp for long-term stimulation, the thermal impacts were maintained similarly over a wide penetration depth range due to more uniform heat transfer from the thermoplasmonic optical fiber. For both conditions, the temperature changes were similar when the penetration depth was beyond $1 \mathrm{~mm}$. Therefore, these effects of the surrounding areas of the fiber can be included to adjust the laser power to implement a consistent effect of localized photothermal stimulation. In addition, we studied the effect of bioheat transfer elements such as cerebral blood flow and metabolic heat generation on the photothermal effect. While these parameters induced changes in the photothermal effect, it was not large enough to significantly control the neural activities. In summary, this study can be an accurate experimental guideline for localized photothermal stimulation using a thermoplasmonic optical fiber in vivo, both for acute and long-term situations.

Author Contributions: Conceptualization, W.H. and H.K.; formal analysis, J.L., D.K., Y.H. and H.J.K.; funding acquisition, H.K.; Investigation, W.H.; methodology, H.-J.K. and J.E.J.; project administration, H.K.; resources, J.E.J;; software, W.H., H.-J.K. and J.E.J.; supervision, H.K.; validation, J.L., D.K., Y.H. and H.-J.K.; writing-original draft, W.H., Y.H. and H.K.; writing—review and editing, W.H., H.-J.K., J.E.J. and H.K. All authors have read and agreed to the published version of the manuscript.

Funding: This work was supported by the DGIST R\&D Program of the Ministry of Science and ICT (19-CoE-BT-03).

Institutional Review Board Statement: Not applicable.

Informed Consent Statement: Not applicable.

Data Availability Statement: Limited data available on request due to the large size of the data.

Conflicts of Interest: The authors declare no conflict of interest. 


\section{References}

1. Passian, A.; Lereu, A.L.; Arakawa, E.T.; Wig, A.; Thundat, T.; Ferrell, T.L. Modulation of multiple photon energies by use of surface plasmons. Opt. Lett. 2005, 30, 41-43. [CrossRef] [PubMed]

2. Lereu, A.L.; Passian, A.; Farahi, R.H.; van Hulst, N.F.; Ferrell, T.L.; Thundat, T. Thermoplasmonic shift and dispersion in thin metal films. J. Vac. Sci. Technol. A Vac. Surf. Film. 2008, 26, 836-841. [CrossRef]

3. Lereu, A.L.; Farahi, R.H.; Tetard, L.; Enoch, S.; Thundat, T.; Passian, A. Plasmon assisted thermal modulation in nanoparticles. Opt. Express 2013, 21, 12145. [CrossRef] [PubMed]

4. Li, C.; Liu, Z.; Yao, P. Gold nanoparticles coated with a polydopamine layer and dextran brush surface for diagnosis and highly efficient photothermal therapy of tumors. RSC Adv. 2016, 6, 33083-33091. [CrossRef]

5. Krishnan, S.; Diagaradjane, P.; Cho, S.H. Nanoparticle-mediated thermal therapy: Evolving strategies for prostate cancer therapy. Int. J. Hyperth. 2010, 26, 775-789. [CrossRef]

6. Huang, X.; Jain, P.K.; El-Sayed, I.H.; El-Sayed, M.A. Plasmonic photothermal therapy (PPTT) using gold nanoparticles. Lasers Med. Sci. 2008, 23, 217-228. [CrossRef]

7. Yoo, S.; Kim, R.; Park, J.H.; Nam, Y. Electro-optical Neural Platform Integrated with Nanoplasmonic Inhibition Interface. ACS Nano 2016, 10, 4274-4281. [CrossRef]

8. Yoo, S.; Hong, S.; Choi, Y.; Park, J.H.; Nam, Y. Photothermal inhibition of neural activity with near-infrared-sensitive nanotransducers. ACS Nano 2014, 8, 8040-8049. [CrossRef]

9. Yizhar, O.; Fenno, L.E.; Davidson, T.J.; Mogri, M.; Deisseroth, K. Optogenetics in Neural Systems. Neuron 2011, 71, 9-34. [CrossRef]

10. Stujenske, J.M.; Spellman, T.; Gordon, J.A. Modeling the Spatiotemporal Dynamics of Light and Heat Propagation for In Vivo Optogenetics. Cell Rep. 2015, 12, 525-534. [CrossRef]

11. Shin, Y.; Yoo, M.; Kim, H.-S.; Nam, S.-K.; Kim, H.-I.; Lee, S.-K.; Kim, S.; Kwon, H.-S. Characterization of fiber-optic light delivery and light-induced temperature changes in a rodent brain for precise optogenetic neuromodulation. Biomed. Opt. Express 2016, 7, 4450-4471. [CrossRef] [PubMed]

12. Spinelli, L.; Zucchelli, L.; Contini, D.; Caffini, M.; Mehler, J.; Fló, A.; Ferry, A.L.; Filippin, L.; Macagno, F.; Cattarossi, L.; et al. In vivo measure of neonate brain optical properties and hemodynamic parameters by time-domain near-infrared spectroscopy. Neurophotonics 2017, 4, 041414. [CrossRef]

13. Baffou, G.; Quidant, R. Thermo-plasmonics: Using metallic nanostructures as nano-sources of heat. Laser Photonics Rev. 2013, 7, 171-187. [CrossRef]

14. Ye, T.; Lai, Y.; Wang, Z.; Zhang, X.; Meng, G.; Zhou, L.; Zhang, Y.; Zhou, Z.; Deng, J.; Wang, M.; et al. Precise Modulation of Gold Nanorods for Protecting against Malignant Ventricular Arrhythmias via Near-Infrared Neuromodulation. Adv. Funct. Mater. 2019, 29, 1902128. [CrossRef]

15. Lee, J.W.; Jung, H.; Cho, H.H.; Lee, J.H.; Nam, Y. Gold nanostar-mediated neural activity control using plasmonic photothermal effects. Biomaterials 2018, 153, 59-69. [CrossRef] [PubMed]

16. Yoo, S.; Park, J.H.; Nam, Y. Single-Cell Photothermal Neuromodulation for Functional Mapping of Neural Networks. Acs Nano 2019, 13, 544-551. [CrossRef]

17. Kang, H.; Lee, G.H.; Jung, H.; Lee, J.W.; Nam, Y. Inkjet-Printed Biofunctional Thermo-Plasmonic Interfaces for Patterned Neuromodulation. ACS Nano 2018, 12, 1128-1138. [CrossRef]

18. Jung, H.; Kang, H.; Nam, Y. Digital micromirror based near-infrared illumination system for plasmonic photothermal neuromodulation. Biomed. Opt. Express 2017, 8, 2866-2878. [CrossRef]

19. Kang, H.; Hong, W.; An, Y.; Yoo, S.; Kwon, H.J.; Nam, Y. Thermoplasmonic Optical Fiber for Localized Neural Stimulation. ACS Nano 2020, 14, 11406-11419. [CrossRef]

20. Kim, S.; Tathireddy, P.; Normann, R.A.; Solzbacher, F. Thermal impact of an active 3-D microelectrode array implanted in the brain. IEEE Trans. Neural Syst. Rehabil. Eng. 2007, 15, 493-501.

21. Das, K.; Mishra, S.C. Simultaneous estimation of size, radial and angular locations of a malignant tumor in a 3-D human breast-A numerical study. J. Biol. 2015, 52, 147-156. [CrossRef]

22. Johannsdotter, S. Design of the Cryocooled DC Discharge System with Heat Transfer Simulations in COMSOL. Master Thesis, Uppsala University, Uppsala, Sweden, 2017.

23. Conroy, D.A.; Spielman, A.J.; Scott, R.Q. Daily rhythm of cerebral blood flow velocity. J. Circadian Rhythm. 2005, 3, 3. [CrossRef]

24. Wells, J.; Kao, C.; Konrad, P.; Milner, T.; Kim, J.; Mahadevan-Jansen, A.; Jansen, E.D. Biophysical mechanisms of transient optical stimulation of peripheral nerve. Biophys. J. 2007, 93, 2567-2580. [CrossRef]

25. Sidor, M.M.; Davidson, T.J.; Tye, K.M.; Warden, M.R.; Diesseroth, K.; McClung, C.A. In vivo optogenetic stimulation of the rodent central nervous system. J. Vis. Exp. 2015, 95, e51483. [CrossRef]

26. Wang, H.; Wang, B.; Normoyle, K.P.; Jackson, K.; Spitler, K.; Sharrock, M.; Miller, C.M.; Best, C.; Llano, D.; Du, R. Brain temperature and its fundamental properties: A review for clinical neuroscientists. Front. Neurosci. 2014, 8, 307. [CrossRef]

27. Lee, J.W.; Kang, H.; Nam, Y. Thermo-plasmonic gold nanofilms for simple and mass-producible photothermal neural interfaces. Nanoscale 2018, 10, 9226-9235. [CrossRef]

28. Fan, B.; Li, W. Miniaturized optogenetic neural implants: A review. Lab Chip 2015, 15, 3838-3855. [CrossRef] 
29. Airan, R.D.; Meyer, R.A.; Ellens, N.P.K.; Rhodes, K.R.; Farahani, K.; Pomper, M.G.; Kadam, S.D.; Green, J.J. Noninvasive Targeted Transcranial Neuromodulation via Focused Ultrasound Gated Drug Release from Nanoemulsions. Nano Lett. 2017, 17, 652-659. [CrossRef]

30. Pasquinelli, C.; Hanson, L.G.; Siebner, H.R.; Lee, H.J.; Thielscher, A. Brain Stimulation Safety of transcranial focused ultrasound stimulation: A systematic review of the state of knowledge from both human and animal studies. Brain Stimul. 2019, 12, 1367-1380. [CrossRef]

31. Tyler, W.J.; Lani, S.W.; Hwang, G.M. Ultrasonic modulation of neural circuit activity. Curr. Opin. Neurobiol. 2018, 50, $222-231$. [CrossRef] 